\title{
Experimental comparison between conventional and hybrid long-range surface plasmon waveguide bends
}

\author{
Aloyse Degiron, ${ }^{1}$ Sang-Yeon Cho, ${ }^{1, *}$ Cameron Harrison, ${ }^{1}$ Nan Marie Jokerst, ${ }^{1}$ Claudio Dellagiacoma, ${ }^{2, \dagger}$ Olivier J. F. Martin, ${ }^{2}$ \\ and David R. Smith, \\ ${ }^{1}$ Center for Metamaterials and Integrated Plasmonics, Department of Electrical and Computer Engineering, Duke University, \\ Box 90291, Durham, North Carolina 27708, USA \\ ${ }^{2}$ Nanophotonics and Metrology Laboratory, Swiss Federal Institute of Technology Lausanne, EPFL-STI-NAM, ELG Station 11, \\ CH-1015 Lausanne, Switzerland
}

(Received 21 November 2007; published 25 February 2008)

\begin{abstract}
We report on the characterization of long-range surface plasmon waveguide bends at telecom wavelengths $(\lambda=1550 \mathrm{~nm})$. The structures consist of a thin Au stripe embedded in a transparent polymer film. When the polymer thickness is larger than the lateral extension of the plasmon, the stripe sustains a conventional long-range mode; in the opposite case, the mode is hybrid because its field distribution is confined by total internal reflection in the dielectric cladding. This hybridization increases the damping by absorption but dramatically reduces the radiation loss that occurs for curved geometries, such as bends. Our results are supported quantitatively by full-wave finite-element simulations.
\end{abstract}

DOI: 10.1103/PhysRevA.77.021804

PACS number(s): 03.50.De, 41.20.Jb, 42.82.Et, 78.67.-n

While metals exhibit large material losses at visible wavelengths, they nevertheless have remarkable optical properties. In particular, the electron density of a metal surface can be coupled with light so as to form a surface plasmon polariton (SPP) — an interface mode characterized by strong local fields and a tremendous sensitivity to its environment [1,2]. The SPP modes supported by thin metal films in a homogeneous dielectric medium are of particular interest because their propagation length can reach several millimeters or more at visible and infrared wavelengths, instead of tens of microns or less as all other SPPs [3-5]. The field distribution of these long-range modes has a large exponential tail extending on each side of the film. Consequently, a significant part of the energy is carried outside the metal, thus minimizing the damping by absorption. Despite their weak optical confinement, long-range SPPs are bound surface modes, so thin metal films can be utilized as efficient low-loss plasmonic waveguides. In order to guide long-range SPPs along a specific direction, the film width must be reduced until the structure becomes a thin metal stripe [6-14]. A variety of components based on the stripe geometry have been designed and fabricated, including interferometers [9], couplers [15], Bragg filters [16], modulators [17,18], and an external cavity laser [19]. Although the material loss in these structures is still considerably larger than in any other integrated waveguides, they might find an application as transitioning elements between purely dielectric structures and short-range SPPs with subwavelength confinement. Metal-based waveguides are also of potential interest for sensing purposes

\footnotetext{
*Present address: Klipsch School of Electrical and Computer Engineering, New Mexico State University, P.O. Box 30001, MSC 3-O, Las Cruces, NM 88003-8001, USA.

†Present address: Laboratoire d'Optique Biomédicale, Swiss Federal Institute of Technology, EPFL-STI-SMT-LOB, BM 5.140, Station 17, CH-1015 Lausanne, Switzerland.

*drsmith@ee.duke.edu
}

because long-range SPPs are very easily perturbed by external changes [12].

In recent years, the interaction between metal stripes and dielectric structures has become the focus of several numerical studies, for example, in the context of directional couplers [20]. A different type of SPP-dielectric interaction occurs for metal stripes embedded in a thin dielectric layer with a refractive index larger than that of the background host [15,21-23]. When the transverse extension of the long-range SPP is larger than the thickness of the dielectric cladding, the mode is hybridized by total internal reflection. In most configurations, the field distribution of the new mode significantly differs from the exponential profile of the unperturbed SPP, which generally results in a more favorable trade-off between optical confinement and propagation length [22,23]. These hybrid modes are especially interesting for curved geometries, for the improved optical confinement considerably mitigates the bend-induced radiation loss while only mildly reducing the propagation length. Therefore, hybrid modes can round sharper bends with losses that can be several tens of $\mathrm{dB}$ smaller than for long-range modes in an infinite environment [22]. In this article, we have verified these predictions experimentally in the near infrared by characterizing the propagation of long-range SPPs along conventional and hybrid $\mathrm{Au} 90^{\circ}$ bends embedded in benzocyclobutene-based (BCB) polymer claddings.

Fabrication of the waveguide samples was performed following the general approach outlined in [8]. Substrates were prepared by first spin-coating a BCB-based resin (Dow $\mathrm{Cy}$ clotene 3022) onto a $\mathrm{Si}$ wafer with 2 microns of $\mathrm{SiO} 2$. The resin was then converted into a solid polymer film by thermal curing in a vacuum oven. In this process, the BCB surface becomes highly planar and smooth, though with poor adhesion properties. We found that it was possible to ensure a good adhesion of the subsequent materials involved in the fabrication process by limiting the $\mathrm{BCB}$ polymerization to about $80 \%$ and by slightly roughening the surface of the film in a plasma asher (Emitech K-1050X). As a next step, the 


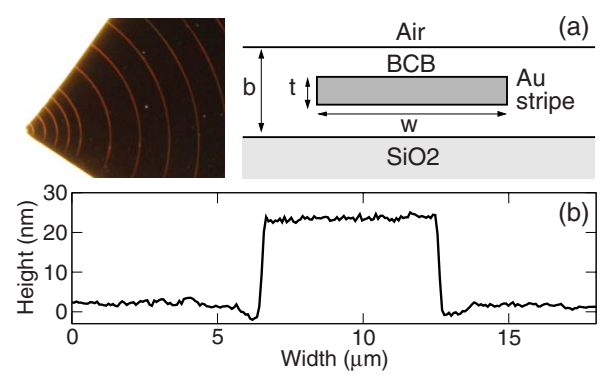

FIG. 1. (Color online) (a) Photograph of an actual set of $90^{\circ} \mathrm{Au}$ bends in BCB (left) and cross-sectional view of the structure (right). (b) Lateral dimensions of a fabricated Au stripe, as measured with our atomic force microscope.

sample was coated with a negative photoresist (Futurrex NR9-1500PY) in which the stripes were transferred from a photomask by contact uv lithography using a Suss MicroTec MJB3 mask aligner operating at $365 \mathrm{~nm}$. A thin Au layer was then deposited on the sample with an electron-beam evaporator (CHA Industries Solution) and the plasmonic waveguides were subsequently revealed by lifting off the remaining photoresist. After covering the stripes with a second thickness of BCB, the whole sample was finally hard cured so as to merge the top and bottom BCB layers. The resulting structures consisted of $90^{\circ}$ bends connected on both sides with a length of straight stripes, although the straights were removed just before the measurements by cleaving the wafer [Fig. 1(a)]. Two thicknesses of BCB, $b=21 \mu \mathrm{m}$ and $b$ $=6.5 \mu \mathrm{m}$, were considered in this study. These values were respectively larger and smaller than the lateral field extension of the mode, meaning that our thick samples supported unperturbed long-range SPPs while our thin samples supported hybrid modes in which the mode field distribution was frustrated at the $\mathrm{SiO} 2$ and air boundaries.

The sample quality was monitored during and after fabrication. In particular, the total BCB thickness was measured by imaging the cleaved facets with an optical microscope. By use of an atomic force microscope [Fig. 1(b)], we established that the metal thickness was uniform across the stripes and that the surface roughness combined with any detection noise was less than $2 \mathrm{~nm}$. We found, however, a very small number of major defects in the width of certain stripes.

Figure 2(a) shows the setup that was used to characterize the structures. The long-range SPPs were excited in an endfire coupling configuration with a single-mode optical fiber coupled to a $1550 \mathrm{~nm}$ laser (Lightwave Measurement System HP 8164A). After propagating along the stripe, the light emitted by the long-range mode was directed toward a pixelized $\mathrm{In}_{x} \mathrm{Ga}_{1-x} \mathrm{As}$ detector array (Sensors Unlimited, SU320MS-1.7RT) mounted on top of a customized Mitutoyo microscope. Long-range SPPs are mostly TM polarized, with the largest magnetic field component parallel to the plane of the stripe so the end-fire coupling efficiency strongly depends on the orientation of the incident field [6,7]. In these experiments, the input polarization has been carefully maintained constant by immobilizing the optical fiber for the time of the measurements. However, this polarization was elliptical, so that a collection of TE-polarized slab modes were

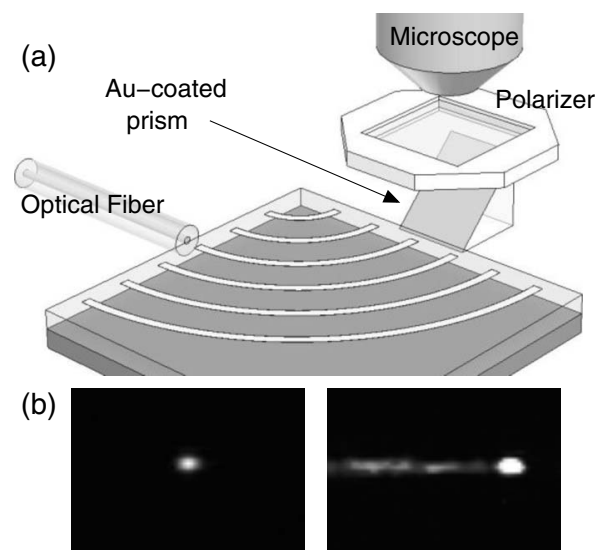

FIG. 2. (a) Experimental setup. The Au-coated prism is used as a mirror to direct the light into the $10 \times$ objective $(0.28$ numerical aperture) of our microscope. (b) Light emitted by a long-range SPP at the output of two $90^{\circ}$ Au bends with $r=7.5 \mathrm{~mm}$ and $r=1 \mathrm{~mm}$, respectively. The image on the right has been saturated for a better visualization of the radiation loss. The image sizes are $\sim 78 \mu \mathrm{m}$ $\times 54 \mu \mathrm{m}$.

excited in the BCB cladding in addition to the SPP modes. The amount of background light collected by our detection apparatus was very small in practice because there was a $90^{\circ}$ angle between the input fiber and the end of the stripe; in addition, a linear polarizer was systematically inserted in front of the microscope objective so as to filter the remaining noise and to ensure that the light originating from the stripe output had the polarization of the long-range plasmon mode.

Figure 2(b) shows the light emitted at the output of two hybrid bends $(b=6.5 \mu \mathrm{m}, t=23 \mathrm{~nm}, w=6.1 \mu \mathrm{m})$ having a radius of curvature equal to $r=7.5 \mathrm{~mm}$ and $r=1 \mathrm{~mm}$, respectively. These images are consistent with previous experimental and numerical studies [9-11,22]; that is, the light originates from a spot centered on the metal stripe that becomes increasingly asymmetric, with an additional tail due to the radiation losses, as the bend becomes sharper. In addition, the intensity pattern for the sharpest bend is clearly limited in the vertical direction due to the finite width of the BCB layer.

The total insertion loss was measured for conventional and hybrid $90^{\circ}$ bends having a metal thickness $t=23 \mathrm{~nm}$ and a width $w=6.5 \mu \mathrm{m}$. The loss is defined as the ratio between the intensity at the output and input of the strip, expressed in $\mathrm{dB}$. To measure the output intensity, we collected the output pattern of the bend and integrated the signal over the emission spot area. A potential difficulty associated with this method arises because part of the light originating from the radiation losses is also collected by our imaging setup, as shown in Fig. 2(b). However, the pattern caused by the radiation loss was consistently found to be much weaker and generally well separated from the main spot by a dark region, so the error made in measuring the intensity is approximately the same as for large radii in the nonradiative limit $( \pm 0.3 \mathrm{~dB})$. While the output intensity is easily measurable with our imaging system, an equivalent measurement of the input intensity is much more difficult due to the uncertainty in the coupling factor between the optical fiber and the stripe. We have therefore normalized our data against an arbitrary 
(a)

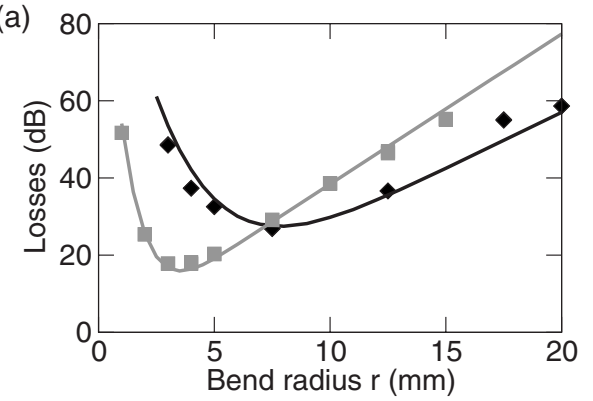

(b)

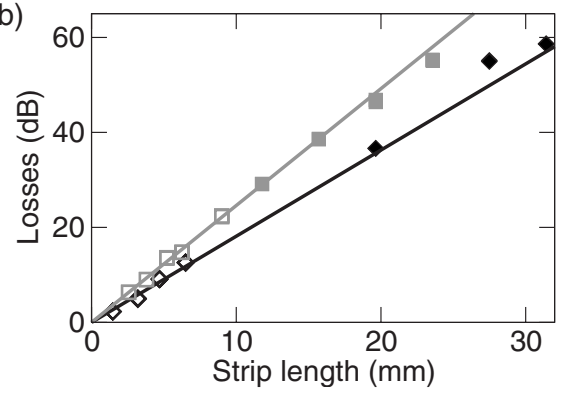

FIG. 3. (a) Insertion loss for two sets of $90^{\circ} \mathrm{Au}$ bends. The black diamonds and line are respectively the measured and simulated loss for the thick sample $(b=21 \mu \mathrm{m}, t=23 \mathrm{~nm}, w=6.5 \mu \mathrm{m})$; the gray squares and line represent the measured and simulation loss for the thin sample $(b=6.5 \mu \mathrm{m}, t=23 \mathrm{~nm}, w=6.1 \mu \mathrm{m})$. (b) Insertion loss vs stripe length for straight and bent long-range SPP waveguides. Again, the black and gray colors have been used to distinguish the thick from the thin BCB samples. The filled and open symbols are the experimental results for large-radii bends and straight stripes, respectively; the lines are the corresponding simulated loss.

constant rather than the true input intensity. We found that the value of this constant is approximately the same as the intensity of the light directly emerging from the fiber. This observation tends to suggest that the coupling factor between the optical fiber and the metal stripe is very high, as in previous experiments with long-range SPPs excited in an endfire configuration [14]. However, a rigorous estimation of this coupling based on the intensity collected at the stripe and fiber outputs is in fact not possible because the numerical aperture of these two waveguides is not the same-and neither is the corresponding fraction of light collected in the solid angle of our experimental apparatus.

Figure 3(a) compares the measured insertion loss for the plasmon waveguide bends as a function of the radius of curvature $r$ with the loss computed from numerical simulations. The computed curves have been generated using an eigenmode solver approach with no adjustable fitting parameters, as described elsewhere [22]. The geometrical parameters used in these simulations were the same as for the actual samples; the $\mathrm{BCB}$ and $\mathrm{SiO} 2$ layers were modeled with a refractive index of 1.535 [8] and 1.444 [9], respectively, and the stripes were assumed to have the bulk permittivity of Au as found in [24] $(\epsilon=-131.95+12.651)$. The curves of Fig. 3(a) have a characteristic shape originally predicted by Berini and Lu for bends in an infinitely thick homogeneous medium [11]. As $r$ increases, the mode tends toward the

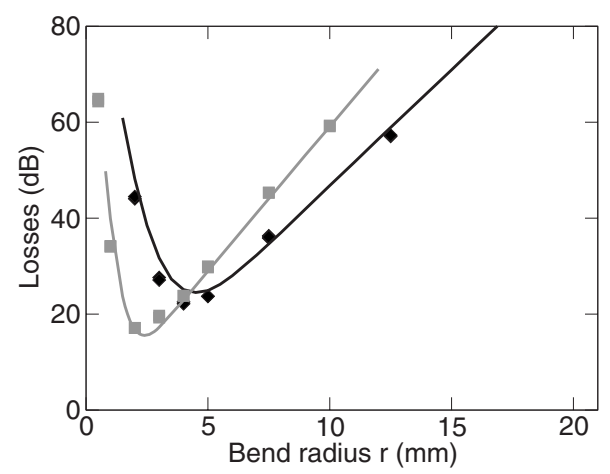

FIG. 4. Insertion loss for two sets of $90^{\circ} \mathrm{Au}$ bends $(w=12 \mu \mathrm{m}$, $t=25.5 \mathrm{~nm})$. The black diamonds and line are, respectively, the experimental points and simulated loss for the thick BCB layer $(b$ $=21 \mu \mathrm{m}$ ); the gray squares and line represent the experimental and simulation loss for the thin BCB layer $(b=6.5 \mu \mathrm{m})$.

bound SPP of a straight waveguide so the asymptote represents the damping by absorption in the metal stripe. We have verified this point experimentally by ensuring that the loss of the corresponding straight waveguide follows the same asymptote when the data are plotted against the total length of the stripe [Fig. 3(b)]. For sharper bends, however, the mode becomes increasingly radiative so the loss eventually increases again despite the smaller length of the bend. Thus, there exists an optimum bend radius $r_{\text {opt }}$ that results from the trade-off between the two types of loss. Figure 3(a) shows that the relative performance of the hybrid and unperturbed modes strongly depends on $r$ : while the propagation length is larger for the unperturbed long-range mode in the straight waveguide limit, the hybrid SPP is much more robust with regard to bending because $r_{\text {opt }}$ is two times smaller and the corresponding loss $10 \mathrm{~dB}$ lower. These trends directly follow from the better confinement of the hybrid mode that increases the absorption but reduces the radiative losses [22].

We have repeated the same experiments for Au stripes with a thickness $t=25.5 \mathrm{~nm}$ and a width $w=12 \mu \mathrm{m}$. Figure 4 shows the experimental and simulated loss for the two thicknesses of BCB considered previously $(b=21 \mu \mathrm{m}$ and $b=6.5 \mu \mathrm{m})$. The curves for the thick and thin samples are less dissimilar than in Fig. 3(a), indicating that the perturbation of the hybrid mode by total internal reflection is not as strong as before. This trend is consistent with the fact that we have increased the lateral dimensions of the stripes, thereby improving the field confinement of the mode [7]. We note that the field confinement is such that the unperturbed mode of Fig. 4 has approximately the same optimum radius $r_{\text {opt }}$ than the hybrid mode of Fig. 3(a). However, enlarging the stripe dimensions leads to higher damping by absorption because the loss at $r_{\text {opt }}$ is $5 \mathrm{~dB}$ higher and the asymptote at large radii is also steeper. In other words, the hybrid mode exhibits a better trade-off between field confinement and loss, a property that arises from its particular field distribution-whereas the field of unperturbed SPPs decreases exponentially away from the surface, the field distribution of hybrid modes has almost a Gaussian shape which allows more energy to be guided outside the metal in a smaller volume [22,23]. 
Finally, a comment should be made about the excellent agreement between the experimental and simulation data of this study. As explained earlier, the only fitting parameter is the input intensity used to normalize the experimental data. All measurements were normalized using the same constant, which means that the absolute vertical position of the experimental curves has been arbitrarily adjusted to match the simulations but that the relative distance between each curve has not been fitted. It is somewhat surprising that the relative vertical position of the experimental curves agrees so well with the simulations because our model does not take into account the coupling between the fiber and the stripes, nor does it include the minor imperfections of the real structures.
This result implies that the propagation is robust to occasional defects in the stripe geometry and also that the coupling between the optical fiber and the different waveguides is approximately constant; that is, it is almost independent of the $\mathrm{BCB}$ thickness, the stripe dimensions, and the radius of curvature. However, we expect that the efficiency of the endfire coupling would eventually change for sharper bends and smaller thicknesses of BCB.

This work was performed under a Multiple University Research Initiative supported by the Air Force Office of Scientific Research (Contract No. FA9550-04-1-0434).
[1] H. Raether, Surface Plasmons (Springer-Verlag, Berlin, 1988).

[2] W. L. Barnes, A. Dereux, and T. W. Ebbesen, Nature (London) 424, 824 (2003).

[3] D. Sarid, Phys. Rev. Lett. 47, 1927 (1981).

[4] J. J. Burke, G. I. Stegeman, and T. Tamir, Phys. Rev. B 33, 5186 (1986).

[5] S. Sidorenko and O. J. F. Martin, Opt. Express 15, 6380 (2007).

[6] R. Charbonneau, P. Berini, E. Berolo, and E. Lisicka-Shrzek, Opt. Lett. 25, 844 (2000).

[7] P. Berini, Phys. Rev. B 61, 10484 (2000).

[8] T. Nikolajsen, K. Leosson, I. Salakhutdinov, and S. I. Bozhevolnyi, Appl. Phys. Lett. 82, 668 (2003).

[9] R. Charbonneau, N. Lahoud, G. Mattiussi, and P. Berini, Opt. Express 13, 977 (2005).

[10] A. Degiron and D. R. Smith, Opt. Express 14, 1611 (2006).

[11] P. Berini and J. Lu, Opt. Express 14, 2365 (2006).

[12] P. Berini, R. Charbonneau, and N. Lahoud, Nano Lett. 7, 1376 (2007).

[13] G. Mattiussi, N. Lahoud, R. Charbonneau, and P. Berini, J. Vac. Sci. Technol. A 25, 692 (2007).

[14] R. Charbonneau, C. Scales, I. Breukelaar, S. Fafard, N.
Lahoud, G. Mattiussi, and P. Berini, J. Lightwave Technol. 24, 477 (2006).

[15] A. Boltasseva, T. Nikolajsen, K. Leosson, K. Kjaer, M. S. Larsen, and S. I. Bozhevolnyi, J. Lightwave Technol. 23, 413 (2005).

[16] S. Jetté-Charbonneau, R. Charbonneau, N. Lahoud, G. Mattiussi, and P. Berini, Opt. Express 13, 4674 (2005).

[17] T. Nikolajsen, K. Leosson, and S. I. Bozhevolnyi, Appl. Phys. Lett. 85, 5833 (2004).

[18] G. Gagnon, N. Lahoud, G. Mattiussi, and P. Berini, J. Lightwave Technol. 24, 4391 (2006).

[19] S. Jetté-Charbonneau and P. Berini, Appl. Phys. Lett. 91, 181114 (2007).

[20] F. Liu, Y. Rao, Y. Huang, W. Zhang, and J. Peng, Appl. Phys. Lett. 90, 141101 (2007).

[21] J. Guo and R. Adato, Opt. Express 14, 12409 (2006).

[22] A. Degiron, C. Dellagiacoma, J. G. McIlhargey, G. Shvets, O. J. F. Martin, and D. R. Smith, Opt. Lett. 32, 2354 (2007).

[23] R. Buckley and P. Berini, Opt. Express 15, 12174 (2007).

[24] W. Lynch and W. R. Hunter, in Handbook of Optical Constants of Solids, edited by E. D. Palik (Academic, New York, 1985). 